\title{
Estudo dos sintomas visuais na síndrome relacionada ao computador e efeitos dos colírios lubrificantes em funcionários do hospital universitário Bettina Ferro de Sousa
}

\author{
Study of visual symptoms in computer syndrome and effects of lubrificating eye drops in \\ employees of the Bettina Ferro de Sousa University Hospital
}

Estudio de los síntomas visuales en el síndrome relacionado al computador y efectos de los colirios lubricantes en funcionarios del Hospital Universitario Bettina Ferro de Sousa

Luiz Ricardo Cruz Neves ${ }^{1 *}$, José Jesu Sisnando D’araujo Filho².

\section{RESUMO}

Objetivo: Estudar os aspectos oculares da Síndrome Visual do Computador (SRVC). Métodos: Estudo com metodologia mista, realizado no Hospital Universitário Bettina Ferro de Sousa (HUBFS), Belém-Pará, em funcionários que utilizavam computador (PC) por pelo menos 2 horas/dia há pelo menos 6 meses, apresentavam ao menos três sintomas da SVRC e não possuíam doenças oftalmológicas. Foram excluídos aqueles que se recusaram a preencher o questionário e/ou não usaram o colírio de forma correta. Os dados foram coletados por meio da apreciação dos questionários, de autoria do pesquisador. $O$ estudo foi realizado com base nas recomendações do Conselho Nacional de Ética em Pesquisa (CONEP). Resultados: A prevalência da síndrome foi de 75,4\% (IC 95\%[64,9\%-85,9\%]). O sexo não demonstrou significância estatística. Variáveis como lluminação artificial inadequada, brilho excessivo da tela, e número de horas/dia em frente ao PC, apresentaram significância estatística na comparação com a SVRC $(p<0,05)$. Sintomas como fadiga, cefaléia e ressecamento, tiveram redução significativa após uso do colírio $(p<0,05)$. Conclusão: A SRVC mostrou-se altamente prevalente na população estudada. Foi comprovada associação diretamente proporcional entre número de horas/dia frente ao $\mathrm{PC}$ e quantidade de sintomas apresentados. $\mathrm{O}$ uso de colírios lubrificantes reduziu os sintomas relatados, assim como melhorou o desempenho no trabalho.

Palavras-chave: Saúde ocular, Transtornos da visão, Medicina do trabalho.

\begin{abstract}
Objective: To study the ocular aspects of Computer Visual Syndrome (SRVC). Methods: A study with a mixed methodology, performed at the Bettina Ferro de Sousa University Hospital (HUBFS), Belém-Pará, in employees who used computer (PC) for an excessive time, had at least three symptoms and didnt have ophthalmic diseases. Those who refused to complete the questionnaire and didnt use the eye drops correctly were excluded. The data were collected through the evaluation of the questionnaires. The study was carried on the recommendations of the National Council of Ethics in Research (NCER). Results: The prevalence of the syndrome was $75.4 \%$ (95\% Cl [64.9\% -85.9\%]). Sex did not show statistical significance. Variables such as inadequate artificial lighting, brightness of the screen, and hours / day in front of the PC, presented statistical significance in the comparison with SVRC $(p<0.05)$. Symptoms such as fatigue, headache and dryness, had significant reduction after use of eye drops $(p<0,05)$. Conclusion: SRVC was highly prevalent in the study population. It was proven a directly proportional association between number of hours / day versus PC and number of symptoms presented. The use of lubricating drops reduced the reported symptoms, as well as improved performance in the work.
\end{abstract}

Key words: Eye health, Vision disorders, Occupational medicine.

\footnotetext{
${ }^{1}$ Médico residente do Hospital Universitário Bettina Ferro de Sousa (HUBFS), Belém - Pará

${ }^{2}$ Médico oftalmologista da Universidade Federal do Pará (UFPA), Belém - Pará.

*E-mail: ricardo.cruz.neves@gmail.com
} 


\section{RESUMEN}

Objetivo: Estudiar los aspectos oculares del Síndrome Visual del Computador (SRVC). Métodos: Estudio em metodología mixta, realizado em el Hospital Universitario Bettina Ferro de Sousa (HUBFS), Belém-Pará, em los pacientes que utilizaban el ordenador por em tiempo excesivo, presentaban al menos tres síntomas de la SVRC, y no tenían enfermedades oftalmológicas. Se excluyeron aquellos que se rehusaron a rellenar el cuestionario y/o no usaron el colirio de forma correcta. La recolección de los datos ocurrió por medio de la apreciación de los cuestionarios. Fue realizado em base em las recomendaciones del Consejo Nacional de Ética em Investigación (CONEI). Resultados: La prevalencia fue del 75,4\% (IC 95\%). El sexo no demostró significancia estadística. Las variables como especie de lluminación, brillo de la pantalla, y número de horas / día frente al ordenador, presentaron significancia estadística $(p<0,05)$. Fatiga ocular, cefalea y sequedad, tuvieron em reducción significativa después del uso del colirio $(p<0,05)$. Conclusión: La SRVC se mostró altamente prevalente em la muestra. Se comprobó em asociación directamente proporcional entre el número de horas / día frente al ordenador y la cantidad de síntomas. El uso de colirios lubricantes redujo los síntomas reportados, como em mejorado el rendimiento em el trabajo.

Palabras clave: Salud ocular, Trastornos de la visión, Medicina del trabajo.

\section{INTRODUÇÃO}

A Síndrome Visual do Computador (SVRC) é definida como a condição resultante do uso excessivo do computador, caracterizada por sintomas visuais, oculares, astenópeicos e musculoesqueléticos, que incluem a fadiga visual, irritação ocular, prurido, olhos secos, lacrimejamento, fotofobia, dor no pescoço e costas, entre outros (BLEHM C et al 2005; ROZA F 2008).

Segundo o Centro de Estudos sobre as Tecnologias da Informação e da Comunicação (CETIC), sob administração do Comitê Gestor da Internet no Brasil, houve um aumento progressivo no nível de informatização no setor privado brasileiro nas últimas décadas. Dados de 2007 mostram que 95\% das empresas investigadas com mais de 10 funcionários possuem computadores, e 92\% contam com alguma forma de conexão à internet (CAVALCANTE CAV e DE SOUZA FN, 2009).

O principal contribuinte dos sintomas da síndrome da visão do computador parece ser o olho seco, resultando em desconforto, distúrbios visuais e instabilidade do filme lacrimal (AGARWAL S et al., 2013).

Fatores ambientais (clima, correntes de ar, iluminação), fatores fisiológicos/físicos (posição da tela do computador, posições ergonômicas da pessoa, brilho da tela do computador) e fatores biológicos (problemas refrativos, olho seco, problemas ortópticos), são considerados determinantes para a aparição da síndrome (BLEHM C et al., 2005).

Estima-se que até $90 \%$ dos usuários de computador por mais de três horas diárias apresentem algum tipo de sintoma relacionado à SVRC. As principias causas estão relacionadas a mecanismos da superfície ocular, acomodativos e extraoculares (AGARWAL S et al., 2013).

Nos Estados Unidos, esta síndrome tornou-se um grande problema de saúde pública, de modo que se estima que $50 \%$ a $75 \%$ das pessoas usuárias de computadores, apresentem sintomas da SVRC. Entretanto, a grande desinformação que existe na população sobre esta síndrome, faz com que diagnosticá-la e trata-la não seja uma tarefa simples (ARRUDA GV et al., 2011).

O presente estudo teve como objetivo geral estudar os sintomas oculares da Síndrome Visual do Computador, assim como avaliar o uso de colírio lubrificante na melhora dos sintomas relacionados à síndrome.

\section{MÉTODOS}

O estudo foi concebido com metodologia mista: por um lado é epidemiológico, observacional e transversal, pois calcula a prevalência da SVRC (amostra inicial, $n=49$ ); por outro lado é de caráter experimental, pois busca avaliar o efeito de uma intervenção (o uso do colírio) sobre um grupo de pacientes (amostra final, $\mathrm{n}=16$ ). 
Foi realizado no Hospital Universitário Bettina Ferro de Sousa (HUBFS), localizado no município de BelémPará. Definiu-se como população de referência os funcionários de ambos os gêneros que estivessem desempenhando suas atividades rotineiras no HUBFS, o que correspondeu a 80 funcionários. $O$ tamanho amostral foi determinado por meio de cálculos estatísticos no programa Bioestat 5.3 , que propôs o número absoluto de 65 indivíduos, com valor de p<0,05 (AYRES M, et al., 2007). A amostra inicial constituiu-se de funcionários que preencheram os critérios de inclusão deste estudo, correspondendo a 49 funcionários. A amostra final foi formada por um subgrupo da amostra inicial, composta dos funcionários que permaneceram até o final da pesquisa, correspondendo a 16 indivíduos. Foram incluídos os funcionários na faixa etária de 18-65 anos, que utilizavam o computador por pelo menos 2 horas/dia há pelo menos 6 meses, apresentavam ao menos três sintomas da SVRC, não possuíam distúrbios visuais (refrativos ou não). Por outro lado, foram excluídos aqueles que se recusaram ou não preencheram o questionário, usaram o colírio de forma inadequada e/ou utilizaram qualquer outra medicação que interferiram nos resultados.

A coleta dos dados aconteceu por meio da análise dos questionários de autoria do pesquisador (Arquivo suplementar). $O$ estudo se deu em duas fases a saber: a primeira realizada através da aplicação destes questionários aos funcionários do hospital (Arquivo suplementar), cujo objetivo foi de identificar os portadores da Síndrome visual relacionado ao computador (SVRC). Após identificar os portadores da SVRC, respeitando os critérios de inclusão e exclusão do estudo, foi fornecido gratuitamente a cada participante da pesquisa, 2 (dois) frascos de colírio lubrificante carmelose sódica $5 \mathrm{mg} / \mathrm{ml}$ (Lacrifilm® $10 \mathrm{ml}$ ), para que os mesmos instilassem uma gota do medicamento em cada olho, de 3 (três) em 3 (três) horas, por um período mínimo de 30 (trinta) dias consecutivos, durante o horário de expediente do trabalho.

Passados os 30 (trinta) dias, estes funcionários foram submetidos à segunda fase da coleta de dados, com a aplicação de um novo questionário (Arquivo suplementar), o qual indagou a respeito dos resultados obtidos após o uso dos colírios lubrificantes neste período de tempo.

A base de dados foi computada e armazenada em uma planilha do software Microsoft Office Excel. Foi previamente fixado o nível de significância alfa $=0.05$ (margem de erro alfa de $5 \%$ ) para rejeição da hipótese de nulidade. A análise estatística dos resultados foi realizado através do software BioEstat, versão 5.3. Foi aplicado o teste Binomial, teste do Qui-quadrado de aderência, teste de Correlação de Spearman, teste t de Student, teste ANOVA (Análise de Variância) com pós-teste de Tukey, teste dos Sinais antes e depois o uso do colírio (AYRES M et al., 2007).

Os gráficos e tabelas foram feitos no Word e Excel 2013. Este estudo foi realizado com base nas recomendações do Conselho Nacional de Ética em Pesquisa (CONEP), que rege as normas para pesquisa com seres humanos. Todos os pacientes assinaram o termo de consentimento livre e esclarecido, indicando que aceitavam participar do estudo. O projeto foi aprovado pelo Comitê de Ética em Pesquisa do Hospital Universitário João de Barros Barreto (HUJBB/UFPA), para execução em 26 de setembro de 2018, sob o número do parecer: 2.919.749 e CAAE: 96008318.3.0000.0017, na Plataforma Brasil.

\section{RESULTADOS}

Foram entrevistados 65 funcionários, dos quais 49 enquadraram-se na SVRC, revelando uma prevalência de 75,4\% (IC 95\% [64,9\%-85,9\%]). Ao longo da pesquisa, 33 funcionários foram excluídos do estudo, restando 16 que participaram de todas as fases da pesquisa. Os resultados a seguir foram organizados em duas partes, compreendidas em:

- AMOSTRA INICIAL, utilizada para o estudo das características da SVRC $(n=49)$;

- AMOSTRA FINAL, utilizada para o estudo dos efeitos do colírio lubrificante sobre os sintomas da síndrome $(n=16)$. 


\section{AMOSTRA INICIAL}

Os resultados a seguir correspondem aos funcionários diagnosticados com a SVRC ( $n=49)$.

O estudo das características dos trabalhadores com a presença de SVRC, mostrou que a média de idade de acometimento era de 37 anos ( $D P= \pm 10,2$ anos). A avaliação da correspondência entre a idade e a quantidade de sintomas foi realizada pela correlação de Spearman, com o $p$-valor $=0.0056^{*}$, mostrando que houve uma relação diretamente proporcional entre a idade e o número de sintomas.

Com relação aos sintomas apresentados, observou-se que fadiga visual $(79,6 \%)$ foi o sintoma mais relatado entre os portadores da SVC, seguido por cefaléia $(77,6 \%)$, embaçamento visual $(63,3 \%)$ e ressecamento/olho seco $(65,3 \%)$ (Tabela 1).

Conforme a análise do Intervalo de Confiança, a frequência dos 6 (seis) principais sintomas relatados (Fadiga, Cefaléia, Embaçamento, ressecamento ocular, Fotofobia e Lacrimejamento) podem atingir e superar a prevalência de $50 \%$ entre as pessoas que apresentam a síndrome (Tabela 1).

Tabela 1 - Ocorrência dos sintomas em n=49 servidores do HUBFS. Belém/PA, ano 2018.

\begin{tabular}{lccc}
\hline & $\mathbf{n}$ & Frequência & IC 95\% \\
\hline Fadiga & 39 & 79.6 & 68.3 a 90.9 \\
Cefaleia & 38 & 77.6 & 65.9 a 89.2 \\
Embaçamento & 31 & 63.3 & 49.8 a 76.8 \\
Ressecamento & 25 & 51.0 & 37.0 a 65.0 \\
Fotofobia & 23 & 46.9 & 33.0 a 60.9 \\
Lacrimejamento & 20 & 40.8 & 27.1 a 54.6 \\
Prurido & 20 & 40.8 & 21.4 a 48.0
\end{tabular}

p-valor $=0.0321^{*}$, teste Binomial para o sintoma prurido.

Fonte: Protocolo de pesquisa, 2018.

Quanto ao gênero, o feminino foi o mais atingido, com uma prevalência de $63,2 \%$ dos que apresentavam a SVRC, seguido por $36,8 \%$ do gênero masculino. Contudo não se observou significância estatística ( $p$ valor = 0,4509) aplicando teste qui-quadrado ao nível de significância 5\% (Tabela 2).

Em relação às características laborais predominantes, as que apresentaram tendência estatisticamente significante foram: Tempo na função de 4 a 8 anos (51\%), pausas durante o uso do computador a cada 30 $60 \mathrm{~min}(55.1 \%)$, Iluminação artificial inadequada (80\%), brilho excessivo $(55.1 \%)$, ausência de ventilação direcionado ao rosto $(77.6 \%)$ e Posição Não-ergonômica (93.9\%). Essas variáveis foram comparadas por meio do teste Qui-quadrado de aderência, mostrando p-valor $<0,05$ (Tabela 2).

A variável "horas de trabalho no computador" apresentou média de $6 \pm 2$ horas. A relação entre essa variável e o número de sintomas foi avaliada pelo teste de Correlação de Spearman, que resultou no p-valor $=0.02389$, mostrando haver proporção de concordância entre as horas no computador e a quantidade de sintomas (Tabela 3).

As horas no computador foram comparadas conforme o sexo do paciente pelo teste $t$ de Student ( $\mathrm{p}$-valor $=0.4509$ ), mostrando não haver associação entre essas variáveis. $O$ número de horas/dia também foi comparado com cada sintoma isoladamente (teste ANOVA - análise de variância), verificando um $p$-valor $=$ 0.4127 . 
Tabela 2 - Caracterização das atividades laborais de $n=49$ servidores do HUBFS. Belém/PA, ano 2018.

\begin{tabular}{|c|c|c|c|}
\hline Característica & $\mathbf{n}$ & $\%$ & p-valor \\
\hline Tempo na Função & & & $0.0119^{*}$ \\
\hline Até 4 anos & 8 & 16.3 & \\
\hline 4 a 8 anos & 25 & 51.0 & \\
\hline 8 ou mais & 16 & 32.7 & \\
\hline Intervalo & & & $<0.0001^{*}$ \\
\hline 30-60 MIN & 27 & 55.1 & \\
\hline 1-2 HORAS & 18 & 36.7 & \\
\hline$>2$ HORAS & 5 & 10.2 & \\
\hline NUNCA & 0 & 0.0 & \\
\hline Ilum. Artificial $(n=35)$ & & & $0.0007^{*}$ \\
\hline Adequada & 7 & 20.0 & \\
\hline Inadequada & 28 & 80.0 & \\
\hline Ilum. Mista $(n=14)$ & 0 & & 0.7893 \\
\hline Adequada & 6 & 42.9 & \\
\hline Inadequada & 8 & 57.1 & \\
\hline Brilho & & & $0.0021^{*}$ \\
\hline Ex. 1 & 7 & 14.3 & \\
\hline Ex. 2 & 15 & 30.6 & \\
\hline Ex. 3 & 27 & 55.1 & \\
\hline Vent. no Rosto & & & $0.0002^{*}$ \\
\hline Sim & 11 & 22.4 & \\
\hline Não & 38 & 77.6 & \\
\hline Posição & & & $<0.0001^{*}$ \\
\hline Ergonômica & 3 & 6.1 & \\
\hline Não ergonômica & 46 & 93.9 & \\
\hline
\end{tabular}

Tabela 3 - Distribuição das Horas no Computador e Número de sintomas da SVC em $n=49$ servidores do HUBFS. Belém/PA, ano 2018

\begin{tabular}{lccc}
\hline & $\mathbf{n}$ & $\%$ & p-valor \\
\hline Horas no computador & & & 0.7672 \\
3 ou 4 & 14 & 28.6 & \\
5 ou 6 & 18 & 36.7 & \\
7 a 10 & 17 & 34.7 & \\
Sintomas & & & $0.0019^{*}$ \\
Três & 18 & 67.3 & \\
Quatro & 17 & 8.2 & \\
Cinco & 3 & 20.4 & \\
Seis & 1 & 4.1 & \\
\hline
\end{tabular}

p-valor $=0.02389$, Correlação de Spearman

Fonte: Protocolo de pesquisa, 2018. 
Em relação ao número de sintomas conforme intervalo durante uso do computador e iluminação, foi observado que os trabalhadores que tinham pausas a cada 30-60 minutos apresentaram menos sintomas (média 3.7 sintomas), com p-valor $=0.0201$, assim como aqueles que utilizavam iluminação mista adequada (média 3.2 sintomas), com p-valor $=0.0059^{*}$ (Tabela 4).

Por outro lado, os indivíduos que tinham hábito de utilizar o brilho do computador do Exemplo 3 (brilho excessivo), apresentaram mais sintomas (média 4.4 sintomas), com p-valor $=0.0338^{*}$ (Tabela 4).

Tabela 4 - Número de Sintomas conforme Intervalo e lluminação em n=49 servidores do HUBFS. Belém/PA, ano 2018.

\begin{tabular}{lccccc}
\hline & \multicolumn{5}{c}{ Quantidade de Sintomas } \\
& $\mathbf{n}$ & Mediana & Média & DP & p-valor \\
\hline Intervalo & & & & & $0.0201^{* *}$ \\
30-60 MIN & 27 & 0 & 3.7 & 0.6 & \\
1-2 HORAS & 18 & 0 & 4.3 & 1.0 & \\
$>$ 2 HORAS & 5 & 0 & 4.2 & 1.1 & \\
llum. Artificial & & & & & $0.0262^{*}$ \\
Adequada & 7 & 2 & 3.4 & 0.5 & \\
Inadequada & 28 & 0 & 4.3 & 0.7 & \\
llum. Mista & & & & & $0.0059^{*}$ \\
Adequada & 6 & 0 & 3.2 & 0.4 & \\
Inadequada & 8 & 1 & 4.3 & 0.7 & \\
Brilho & & & & & $0.0338^{* *}$ \\
Ex. 1 & 7 & 0 & 3.9 & 0.7 & \\
Ex. 2 & 15 & 0 & 3.7 & 0.8 & \\
Ex. 3 & 27 & 0 & 4.4 & 0.8 & \\
\hline * & & 0 &
\end{tabular}

*teste $t$ de Student; ${ }^{* *}$ ANOVA com pós-teste de Tukey.

Fonte: Protocolo de pesquisa, 2018.

\section{AMOSTRA FINAL}

Os resultados descritos abaixo correspondem aos funcionários que participaram de todas as fases do estudo e que respeitaram todos os critérios pré-estabelecidos desta pesquisa.

Dos 16 trabalhadores que foram avaliados sob efeito do colírio, $25 \%$ eram do sexo masculino (4 trabalhadores) e $75 \%$ (12 trabalhadores) eram do sexo feminino.

Tabela 5 - Ocorrência dos sintomas em n=16 servidores do HUBFS. Belém/PA, ano 2018.

\begin{tabular}{lcccccc}
\hline \multicolumn{1}{c}{ Sintomas } & $\mathbf{n}$ & Antes & Depois & A - D & \\
\hline Fadiga & $\mathbf{n}$ & $\%$ & $\mathbf{n}$ & $\%$ & $\%$ & p-valor \\
Lacrimejamento & 7 & 81.3 & 5 & 31.3 & 50.0 & $0.0101^{*}$ \\
Fotofobia & 9 & 43.8 & 5 & 31.3 & 12.5 & 0.2501 \\
Prurido & 8 & 56.3 & 5 & 31.3 & 25.0 & 0.1542 \\
Embaçamento & 9 & 50.0 & 5 & 31.3 & 18.7 & 0.3802 \\
Ressecamento & 10 & 62.5 & 3 & 18.8 & 43.7 & $0.0313^{*}$ \\
Cefaléia & 12 & 75.0 & 3 & 18.8 & 56.2 & $0.0195^{*}$ \\
\hline
\end{tabular}

*Teste t de Student: Variação percentual entre Antes e Depois.

Fonte: Protocolo de pesquisa, 2018. 
Quanto a variação dos sintomas, observou-se que o efeito do colírio foi significativamente ( $p$-valor $\left.<0.05^{\star}\right)$ observado nos seguintes sintomas: Cefaléia $\left(p=0.0020^{*}, A-D=50 \%\right)$, Fadiga $(p=0.0101, A-D=50 \%)$, Ressecamento ( $\left.p=0.0195^{\star}, A-D=43.7 \%\right)$ e Embaçamento $\left(p=0.0313^{\star}, A-D=31.3 \%\right)$ (Tabela 5).

Por fim, pode-se observar que $75 \%$ dos funcionários $(n=12)$, referiram melhora no desempenho laboral após o período de aplicação do colírio.

\section{DISCUSSÃO}

Neste trabalho foi evidenciada uma maior prevalência em indivíduos do gênero feminino $(63,2 \%)$. Resultados semelhantes foram encontrados por ROZA F (2008), que demonstrou o predomínio de mulheres em um estudo que verificou a presença da síndrome em operadores de tele atendimento. BREWER S (2006), descreve que nos países industrializados é observada a forte presença de mulheres no setor: em média, 7 a cada 10 operadores. SÁ EC (2010), também evidenciou em sua pesquisa uma prevalência de astenopia ocupacional mais elevada no gênero feminino $(56,7 \%)$.

Entre os resultados, encontrou-se que os hábitos no uso do computador, como número de horas/dia no uso do computador (média de $6 \pm 2$ horas), e utilização de brilho excessivo na tela $(55,1 \%)$, aumentam a quantidade e a frequência das queixas visuais, o que concorda com ROSENFIELD M (2011) e BLEHM C et al. (2005), que aponta que trabalhar mais de quatro horas seguidas aumenta o risco de desenvolver a SVRC.

CAVALCANTE CAV E SOUZA SN (2009), também constataram que as queixas visuais foram mais frequentes em operadores que trabalharam de 6 a 9 horas por dia (75\%). De maneira semelhante, NASCIMENTO CAV (2004), afirmou que 90\% dos que trabalham mais de 2 horas diárias apresentam sintomas visuais e os que gastam mais de 4 horas por dia experimentam os sintomas com duração e gravidades maiores.

BALI J et al. (2007) citaram que $90 \%$ dos trabalhadores americanos, que utilizaram computador por mais de 3 horas por dia, experimentaram os sintomas da SVRC de alguma forma. BLEHM C et al. (2005) afirma que o trabalho contínuo frente ao monitor de computador por mais de 4 horas diárias é um risco para apresentar astenopia.

Os sintomas da SVRC mais prevalentes na amostra foram fadiga visual $(79,6 \%)$, seguido por cefaléia $(77,6 \%)$, embaçamento visual $(63,3 \%)$ e ressecamento $(65,3 \%)$, concordando assim com os sintomas predominantes descritos na literatura (BLEHM C et al., 2005; SCHAEFFER TMC 2007; SHEEDY JE et al., 2004; WOLKOFF P 2005). Segundo BLEHM C et al. (2005), o principal contribuinte dos sintomas da síndrome da visão do computador parece ser o olho seco, que resulta em desconforto, distúrbios visuais e instabilidade do filme lacrimal. Contribuindo para isso, GENTIL RM et al. (2011) afirma que, com o envelhecimento, ocorre diminuição de volume e fluxo lacrimal, hiperosmolaridade, perda da estabilidade do filme lacrimal e alteração na composição lipídica da glândula de Meibomius, facilitando o desenvolvimento do olho seco.

De acordo com esta pesquisa, a fadiga visual prevaleceu sobre os outros sintomas da síndrome $(79,6 \%)$. Para BLEHM C et al. (2005), a fadiga visual é o sintoma mais frequente nos trabalhadores que ficam mais de 4 horas diárias frente ao monitor. CAVALCANTE E SOUZA (2009) também observaram que o sintoma mais relatado era a fadiga visual acompanhado da cefaleia, que foi o segundo sintoma mais prevalente encontrado neste estudo $(75,5 \%)$.

As características laborais predominantes entre os servidores que apresentaram a SVRC foram: tempo na função acima 4 anos, iluminação artificial inadequada, brilho excessivo (exemplo 3) e posição nãoergonômica. Segundo GENTIL RM et al. (2011), fatores relacionados ao desconforto e à fadiga visual estão relacionados com os fatores ergonômicos. Entre eles, a iluminação é uma das causas que têm influência significativa nos danos aos olhos, sendo que uma distribuição errada de luminância pode levar à fadiga ocular (GENTIL RM et al., 2011). Estudos sugerem que a distância superior a $40 \mathrm{~cm}$ dos olhos em relação à tela, podem resultar em menor número de queixas e menos esforço visual. Da mesma maneira, a tela posicionada ao mesmo nível dos olhos, assegura menor cansaço visual (NASCIMENTO CAV, 2004). 
Os trabalhadores que apresentam pausas durante o uso do computador entre 30-60 minutos, e trabalhavam em um ambiente com iluminação mista adequada, apresentaram menos sintomas. BLEHM C et al. (2005), afirma que longos períodos de trabalho sem pausas são prejudiciais para os sintomas oculares, sendo que intervalos frequentes são recomendados para restaurar e relaxar o sistema de acomodação e evitar a fadiga ocular. Acredita-se que desviar o olhar para um objeto distante pelo menos duas vezes por hora durante o uso do computador seja suficiente para a prevenção da fadiga visual (GENTIL RM et al., 2011).

Neste estudo, verificou-se que houve melhora significativa dos sintomas visuais após a aplicação do colírio lubrificante. SKILLING FC (2005), observou que o uso dos colírios lubrificantes mostraram-se eficazes no tratamento de sintomas visuais em pessoas com SVRC.

É de suma importância que haja um consenso da comunidade cientifica internacional, com o objetivo de definir os sinais e sintomas que compõem a síndrome e suas variações. Da mesma forma, é necessário estabelecer um protocolo que sirva de instrumento para diagnosticar e tratar os pacientes que apresentem a SVRC.

Para isso, faz-se necessário outras pesquisas com o intuito de se construir um quadro da real situação da saúde visual dos trabalhadores que utilizam computadores, que permitirá estabelecer a real prevalência da síndrome na população exposta.

Por fim, este estudo aponta a necessidade de ações nas situações laborais, envolvendo modificações das condições do ambiente e organização do trabalho, análise da atividade profissional e, ao mesmo tempo, avaliação das características dos trabalhadores.

\section{CONCLUSÃO}

Os funcionários do HUBFS-PA podem ser considerados uma população com uma alta prevalência da SVRC, visto que na amostra encontrou-se a síndrome em mais da metade dos participantes $(75,4 \%)$. Os colírios lubrificantes mostram-se eficazes em reduzir os sintomas dos pacientes com a SRVC.

\section{AGRADECIMENTOS}

Á empresa GENOM, através da representante Joyce Oliveira da Cruz Rocha, a qual disponibilizou gratuitamente os colírios lubrificantes (LACRIFILM®) que foram ofertados aos indivíduos que participaram desta pesquisa.

Os autores declaram não haver conflito de interesse que poderia se constituir em um impedimento para a publicação deste artigo.

\section{ARQUIVOS SUPLEMENTARES}

Arquivos suplementares disponíveis no sítio de registro DOI: https://doi.org/10.25248/reas.e591.2019

\section{REFERÊNCIAS}

1. ARRUDA GV et al. Dry eye: etiopathogenesis and treatment. Arq Bras oftalmol, v.73, n.2, p.197-203. Abr. 2011.

2. AGARWAL $S$ et al. Evaluation of the Factors which Contribute to the Ocular Complaints in Computer Users. Journal of clinical and diagnostic research: JCDR, v. 7, n. 2, p. 331-335, fev. 2013.

3. ANSHEL JR. Visual ergonomics in the workplace. AAOHN journal: official journal of the American Association of Occupational Health Nurses, v. 55, n. 10, p. 414-420; quiz 421-422, out. 2007.

4. AYRES M et al.. BioEstat 5.3: Aplicações Estatísticas nas Áreas das Ciências Biológicas e Médicas. 5. ed. Belém-PA: Publicações Avulsas do Mamirauá, 2007. 
5. BALI $\mathrm{J}$ et al. Computer vision syndrome: A study of the knowledge, attitudes and practices in Indian Ophthalmologists. Indian Journal of Ophthalmology, v. 55, n. 4, p. 289, 2007.

6. BLEHM C et al. Computer Vision Syndrome: A Review. Survey of Ophthalmology, v. 50, n. 3, p. 253-262, maio 2005.

7. BREWER S. Workplace interventions to prevent musculoskeletal and visual symptoms and disorders among computer users: a systematic review. Journal of occupational rehabilitation, v. 16, n. 3, p. 325-358, set. 2006.

8. CAVALCANTE CAV, DE SOUZA FN. Efeito do uso dos colírios lubrificantes na síndrome relacionada a computador. Trabalho de conclusão de curso. Brasil, Pará, 2009.

9. CINGU AK et al. Evaluation of Corneal Parameters with Scheimpflug Imaging in Patients with Rheumatoid Arthritis. Ocular immunology and inflammation, 4 jun. 2013.

10. FONSECA C. História da Computação. O caminho do pensamento e da tecnologia. 1ra. ed. Porto Alegre, 2007.

11. GENTIL RM et al. Síndrome da visão do computador. Science in Health, v.2, n.1, p.64-66, jan-abril 2011.

12. ROJAS JG. Alteraciones Acomodativas. Imagen Optica, v. 7, p. 20-26, out. 2005.

13. ROSENFIELD M. Computer vision syndrome: a review of ocular causes and potential treatments. Ophthalmic and Physiological Optics, v. 31, n. 5, p. 502- 515, 2011.

14. ROZA F. A influencia da altura da tela do computador na ocorrencia da dor cervical. Campinas: Universidade Estadual de Campinas, 30 set. 2008.

15. SÁ EC. Fatores de risco para a síndrome visual associada ao uso do computador em operadores de duas centrais de teleatendimento em São Paulo, Brasil; Universidade de São Paulo. Faculdade de Saúde Pública. Departamento de Saúde Ambiental, 2010.

16. SCHAEFER TMC. Análise das alterações do piscar, do filme lacrimal e da superfície ocular induzidas pelo uso de monitor de computador. Tese de Doutorado, 2007.

17. SHEEDY JE et al. Is all asthenopia the same? Optometry and vision science: official publication of the American Academy of Optometry, v. 80, n. 11, p. 732-739, nov. 2003.

18. SHEEDY JE et al. Visual effects of the luminance surrounding a computer display. Ergonomics, v. 48, n. 9 , p. 1114-1128, 2005.

19. SKILLING FC. Effects of two eye drop products on computer users with subjective ocular discomfort. Optometry, v.76, n.1, p. 47-54, January. 2005.

20. WOLKOFF P. Eye complaints in the office environment: precorneal tear film integrity influenced by eye blinking efficiency. Occupational and Environmental Medicine, v. 62, n. 1, p. 4-12, jan. 2005. 